\title{
NUEVOS REGISTROS Y AMPLIACIONES DE ÁMBITO GEOGRÁFICO PARA LAS ARAÑAS (ARACHNIDA: ARANEAE) DE LA HISPANIOLA, ANTILLAS MAYORES
}

\author{
Alexander Sánchez-Ruiz \\ Centro Oriental de Ecosistemas y Biodiversidad (BIOECO). Museo de Historia Natural “Tomás Romay”. José A. \\ Saco \# 601, Santiago de Cuba 90100, Cuba. alex@bioeco.ciges.inf.cu

\section{RESUMEN}

A partir de la identificación, catalogación y ordenamiento de la colección de arañas del Museo Nacional de Historia Natural, Santo Domingo (MNHNSD) y la revisión de material adicional procedente de otras colectas en República Dominicana, se registran por primera vez para La Hispaniola cuatro géneros con representantes en América Central y Las Antillas, pero desconocidos para esta isla: Masteria L. Koch, 1873 (Dipluridae), Strotarchus Simon, 1888 (Miturgidae), sólo conocidos de la fauna fósil, Lygromma Simon, 1893 (Prodidomidae) y Peckhamia Simon, 1901 (Salticidae). Con estos nuevos hallazgos la fauna de arañas de La Hispaniola se eleva a 310 especies agrupadas en 43 familias. Se amplía también el ámbito geográfico de otras tres especies endémicas: Wulfila gracilipes (Banks, 1903) (Anyphaenidae), Mimetus hispaniolae Bryant, 1948 (Mimetidae) y Ariadna multispinosa Bryant, 1948 (Segestriidae). Las dos primeras conocidas únicamente de la localidad tipo.

Palabras clave: nuevos registros, arañas, La Hispaniola, Antillas Mayores.

\section{ABSTRACT}

As a result of the identification and cataloguing of the spiders collection of the Museo Nacional de Historia Natural, Santo Domingo (MNHNSD) and the revision of additional material from the Dominican Republic, four genera occurring in Central America and the West Indies but unknown for Hispaniola Island are reported as new for this island: Masteria L. Koch, 1873 (Dipluridae), Strotarchus Simon, 1888 (Miturgidae), only known from the fossil fauna, Lygromma Simon, 1893 (Prodidomidae) and Peckhamia Simon, 1901 (Salticidae). The finding of these new records elevates to 310 species and 43 families of spiders reported for Hispaniola Island. The geographic limits of other three endemic species: Wulfila gracilipes (Banks, 1903) (Anyphaenidae), Mimetus hispaniolae Bryant, 1948 (Mimetidae) and Ariadna multispinosa Bryant, 1948 (Segestriidae) are enlarged. The two first ones were known only from the type locality.

Key words: new records, spiders, Hispaniola, Greater Antilles.

\section{INTRODUCCIÓN}

Desde finales del siglo XIX se inician los estudios sobre la fauna actual de arañas de La Hispaniola (Haití y República Dominicana). La primera araña endémica de esta isla fue descrita por el alemán A. Ausserer (1875) como Cyrtosternum cursor, hoy bajo el género Cyrtopholis, a partir de una hembra de Theraphosidae procedente de Santo Domingo. Posteriormente, Simon $(1888,1895,1903)$ describe y registra varias especies más para la isla, fundamentalmente de las familias Theraphosidae, Araneidae y Salticidae. La primera contribución enfocada únicamente en las arañas de La Hispaniola fue realizada por N. Banks (1903), registrando 63 especies para Haití.

En esa época, otros autores como Strand $(1909,1916)$; Petrunkevitch $(1911,1928)$ y Gertsch $(1941)$ registraron en sus obras especies para La Hispaniola. Sin embargo, las mayores contribuciones hasta la fecha corresponden a Bryant $(1943,1945,1948)$, quien describe más de 100 especies y registra varias decenas para esta isla. Luego de los trabajos de Bryant, los estudios posteriores sobre las arañas actuales de La Hispaniola se basaron sólo en descripciones y registros de especies en grandes obras de revisiones a nivel genérico, fundamentalmente de las arañas de Norteamérica, América Central y Las Antillas. La riqueza de especies de la isla se fue incrementando entonces a partir de la revisión de Selenopidae por Muma (1953), quien describe dos especies endémicas del género Selenops, y Levi (1954, 1955, 1957, 1959, 1964, 1963a, 1963b, 1977, 1986, 1991a, 1991b, 1995, 1999, 2001), quien de forma sistemática hace la revisión de varios géneros neotropicales de Theridiidae, Araneidae y Tetragnathidae, describiendo y 
registrando una parte de las arañas actuales de La Hispaniola. Con estos estudios contribuyeron además Exline \& Levi (1962) y Berman \& Levi (1971), quienes revisaron los géneros Argyrodes (Theridiidae) y Neoscona (Araneidae) respectivamente. Chickering (1967) revisa el género Nops describiendo una nueva especie para La Hispaniola. Platnick \& Shadab $(1974,1980)$ describen en sus obras tres especies de Trachelas (Corinnidae) y una de Cesonia (Gnaphosidae). Finalmente, con la revisión del género Loxosceles de Gerstch \& Ennik (1983) la fauna de Sicariidae de la isla queda establecida.

Afortunadamente, a partir de 1992, con los trabajos publicados por Alayón García (1992, 1995b, 2002a, 2004, 2007) y Alayón García et al. (2001), estos estudios vuelven a tomar un carácter más específico, enfocados particularmente en la fauna de arañas de la isla. Paralelamente, Coyle (1995); Penney (1999, 2004); Huber (2000); Penney \& Pérez-Gelabert (2002); Sánchez-Ruiz (2005); Huber \& Wunderlich (2006) y Hormiga et al. (2007) describen y registran nuevas especies y familias para La Hispaniola incrementando el conocimiento de la fauna actual de arañas de la isla.

Recientemente, durante la identificación, catalogación y ordenamiento de la colección de arácnidos del Museo Nacional de Historia Natural de Santo Domingo (MNHNSD) fue posible el hallazgo de cuatro nuevos registros de arañas para La Hispaniola y la ampliación del ámbito geográfico de otras tres especies endémicas, dos de las cuales se conocían únicamente de la localidad tipo. Estos hallazgos son presentados en este trabajo.

\section{MATERIALES Y MÉTODOS}

Durante los meses de octubre, noviembre y diciembre de 2007 se realizó la identificación, catalogación y ordenamiento de la colección de arácnidos del Museo Nacional de Historia Natural, Santo Domingo, República Dominicana (MNHNSD). Se revisaron un total de 884 lotes que contenían 3,545 especímenes pertenecientes a 125 especies, 78 géneros y 35 familias. Se examinó además material adicional del Centro Oriental de Ecosistemas y Biodiversidad (BSC.AR, BIOECO) procedente de varios viajes de colecta desarrollados en la República Dominicana. El material fue examinado utilizando un microscopio estereoscópico MEIJI TECHNO Modelo EMZ-5TR con aumento hasta 45X. Todo el material examinado se encuentra depositado en las colecciones del MNHNSD y BSC.AR (BIOECO).

\section{RESULTADOS}

\section{NUEVOS REGISTROS PARA LA HISPANIOLA}

Familia Dipluridae

Masteriasp.

Material examinado: $2 \uparrow$ MNHNSD. 09.561. Engombe, Distrito Nacional, República Dominicana. 22 de noviembre, 2007. G. de los Santos. En la hojarasca.

Familia Prodidomidae

Lygromma chamberlini Gertsch, 1941

Material examinado: 1 q MNHNSD. 09.559. Guaraguao, La Altagracia, Parque Nacional del Este, República Dominicana. (camino a las cavernas). 6 de mayo, 1988. J. Infante.

Familia Salticidae

Peckhamia americana (Peckham y Peckham, 1892)

Material examinado: $3 q 2 \widehat{\jmath}$ MNHNSD. 09.180. Norte de Isla Catalina, La Romana, República Dominicana. 25 de enero, 1992. K. Guerrero.

Familia Miturgidae

Strotarchus sp.

Material examinado: 1 q MNHNSD. 09.558. 3 km. E de las Mercedes, Pedernales, República Dominicana. 900 pies snm. 20 de junio, 1989. D. Matusik y K. Guerrero. Bajo piedra. 


\section{AMPLIACIÓN DE ÁMBITO GEOGRÁFICO}

Familia Anyphaenidae

Wulfila gracilipes (Banks, 1903)

Material examinado: 2 \% 11 juveniles MNHNSD 09.211-07. Boca de Yuma, San Rafael de Yuma, Provincia La Altagracia, Parque Nacional del Este, República Dominicana. Lat.: $18^{\circ} 21^{\prime} 35^{\prime \prime}$ N. Long.:68 37'10" W. 26 de marzo, 2002. B. Farrel y K. Guerrero. Tipo de colecta: fumigado de canopia. 1 BSC.AR 534. Engombe, Distrito Nacional, República Dominicana. 22 de noviembre, 2007. A. Sánchez.

Registros anteriores: Puerto Príncipe, Haití (Banks, 1903).

Familia Mimetidae

Mimetus sp.

Material examinado: 1 MNHNSD 09.557-08. Boca de Yuma, San Rafael de Yuma, Provincia La Altagracia, Parque Nacional del Este, República Dominicana. 26 de marzo, 2002. B. Farell y K. Guerrero. Lat. $18^{\circ} 21^{\prime} 35^{\prime \prime} \mathrm{N}$. Long. $68^{\circ} 37^{\prime} 10^{\prime \prime} \mathrm{W}$. Tipo de colecta: fumigado de canopia. 1 9 MNHNSD 09.122-07. Los Borbollones, La Descubierta. Provincia Independencia, República Dominicana. 30 de enero, 1991. Félix del Monte.

Registros anteriores: Loma Rucilla, Cordillera Central, República Dominicana (Bryant, 1948).

Mimetus hispaniolae Bryant, 1948

Material examinado: 10 MNHNSD 09.540-08. Pedernales, km. 25 de la carretera Alcoa-Sierra de Bahoruco (en una finca), República Dominicana. 20 de mayo, 1992. Tipo de colecta: red de golpeo, en el día, a $28^{\circ} \mathrm{C}$.

Registros anteriores: Bosque cerca de Valle Nuevo, Cordillera Central, República Dominicana (Bryant, 1948).

\section{Familia Segestriidae}

\section{Ariadna multispinosa Bryant, 1948}

Material examinado: 1 MNHNSD 09.89-07. Pajón Blanco, Constanza, Provincia La Vega, República Dominicana. 13 de mayo, 1998. 1 juvenil MNHNSD 09.164-07. Boca de Yuma, San Rafael de Yuma, Provincia La Altagracia, Parque Nacional del Este, República Dominicana. Lat.: $18^{\circ} 21^{\prime} 35^{\prime \prime}$ N. Long.: $68^{\circ}$ 37'10" W. 26 de marzo, 2002. B. Farrel y K. Guerrero. Tipo de colecta: fumigado de canopia. 3 q MNHNSD 09.90-07. 1 o 2 km. W de La Gran Chorra, San Rafael de Yuma, Provincia La Altagracia, Parque Nacional del Este, República Dominicana. 2 de mayo, 1992. Félix del Monte. 3 q BSC.AR 343. Km 6 Carretera Oviedo-Pedernales, Provincia de Pedernales, República Dominicana. 1de febrero, 2005. A. Sánchez. Bajo corteza de árbol.

Registros anteriores: Loma Rucilla, Pico del Yaque, República Dominicana (Bryant, 1948). Bosque cerca de Valle Nuevo, Cordillera Central, República Dominicana (Bryant, 1948).

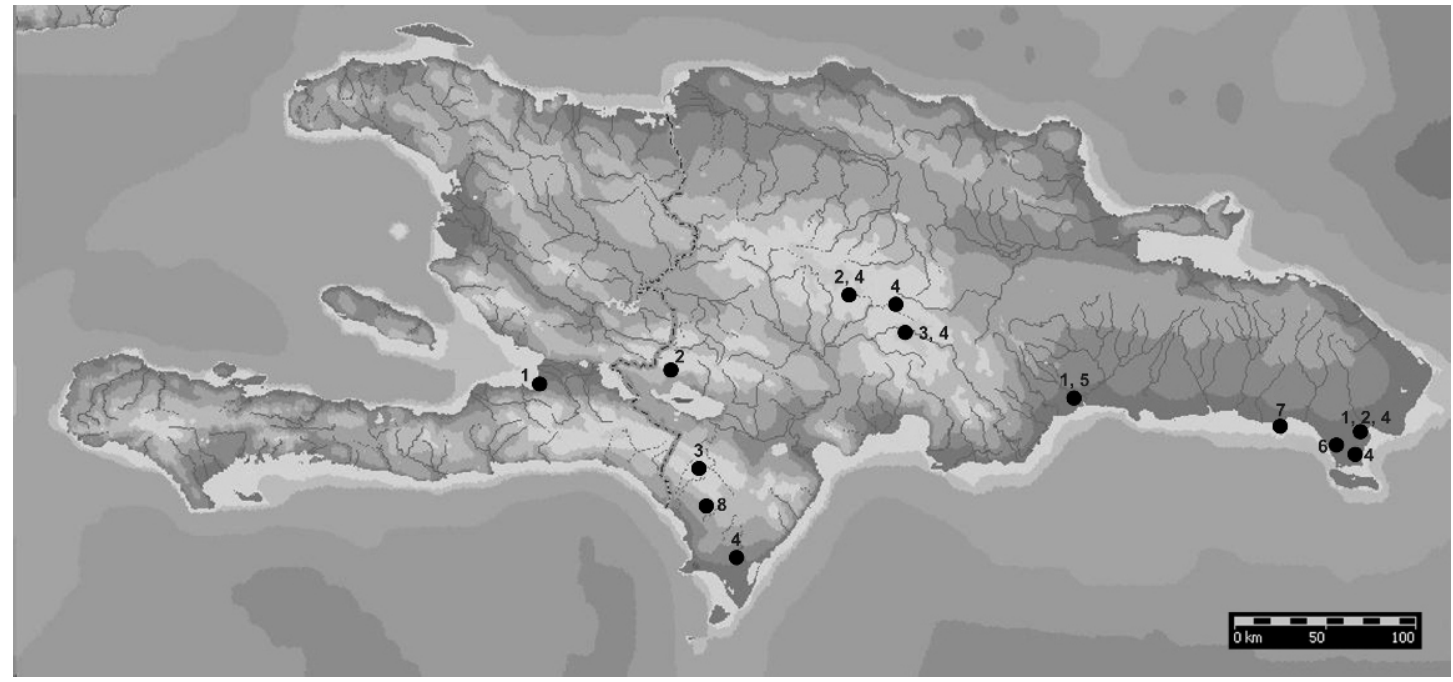

Figura 1. Localidades de colecta. Wulfila gracilipes (Banks, 1903) (1), Mimetus sp. (2), Mimetus hispaniolae Bryant, 1948 (3), Ariadna multispinosa Bryant, 1948 (4), Masteria sp. (5), Lygromma chamberlini Gertsch, 1941 (6), Peckhamia americana (Peckham \& Peckham, 1892) (7) y Strotarchus sp. (8). Mapa adaptado de Microsoft Encarta 2006. 


\section{DISCUSIÓN}

El hallazgo de cuatro nuevos registros de géneros para La Hispaniola incrementa la riqueza de arañas de esta isla a 310 especies agrupadas en 43 familias. Estos nuevos registros poseen representantes en mayor o menor medida en las Antillas. El género Masteria L. Koch, 1873 está representado en esta área por siete especies distribuidas en Cuba (2), Jamaica (2), Puerto Rico (1), San Vicente (1) y Trinidad (1), todas endémicas de sus respectivas islas (Platnick, 2007). Atendiendo a la distribución y endemismo del género, era de esperar el descubrimiento de representantes de Masteria en La Hispaniola, los cuales podrían tratarse incluso de una especie nueva.

La especie Lygromma chamberlini Gertsch, 1941, se conocía anteriormente del Sureste de Panamá (Platnick y Shadab, 1976). Posteriormente Alayón García (1995a) la registra del Este de Cuba constituyendo el primer registro para las Antillas. De igual forma Peckhamia americana (Peckham y Peckham, 1892) se conocía de los Estados Unidos y México (Peckham y Peckham, 1909), y en las Antillas únicamente de Cuba (Alayón García, 2002b). El hallazgo de estas dos especies en La Hispaniola refuerza los criterios sobre la estrecha relación biogeográfica entre esta isla y el este del archipiélago cubano.

El género Strotarchus Simon, 1888 está representado en las Antillas por Strotarchus nebulosus Simon, 1888 registrado por Alayón García (2002b) de Cuba. De los cuatro nuevos registros, sólo Strotarchus y Masteria se conocen de representantes fósiles en La Hispaniola (Penney y Pérez-Gelabert, 2002; Penney, 2004) con las especies Strotarchus heidti Wunderlich, 1988 y Masteria sexoculata (Wunderlich, 1988), ambas conocidas del ámbar del Mioceno de la República Dominicana.

Por otra parte, la especie Mimetus hispaniolae Bryant sólo se conocía del macho holotipo descrito hace más de 50 años de un bosque cerca de Valle Nuevo, Cordillera Central, República Dominicana. Otro macho fue localizado en la Provincia de Pedernales aumentando el ámbito de distribución de la especie a la paleoisla sur. Bryant (1948) supone que una hembra juvenil colectada en Loma Rucilla, Cordillera Central, República Dominicana pertenece a esta especie. Dos hembras examinadas, procedentes de la Provincia Independencia y de la Provincia La Altagracia, posiblemente también pertenezcan a esta especie.

Con este trabajo se amplía el ámbito geográfico de otras dos especies (Fig. 1). Ariadna multispinosa descrita por Bryant (1948) no se había vuelto a registrar de otras localidades en La Hispaniola. El hallazgo de esta especie en las provincias de La Vega, La Altagracia y Pedernales demuestra que posee una distribución más amplia de lo que se conocía. Wulfila gracilipes descrita hace más de un siglo para Puerto Príncipe en Haití, no se conocía de la República Dominicana.

\section{AGRADECIMIENTOS}

Este trabajo fue realizado gracias al Museo Nacional de Historia Natural, Santo Domingo (MNHNSD), la Universidad Autónoma de Santo Domingo (UASD) y el Consorcio Ambiental Dominicano (CAD), que aportaron los fondos para mi trabajo en la República Dominicana. Especial agradecimiento a cada una de las personas del MNHNSD por su valiosa ayuda durante los trabajos desarrollados en esa institución. Particularmente a Celeste Mir y Carlos Suriel por toda su preocupación y apoyo, también a los técnicos del Departamento de Ciencias de esta institución: Elvi de los Santos, por los dibujos realizados de los nuevos registros, Kennida Polanco, Solanlly Carrero y Gabriel de los Santos por su ayuda en la identificación, separación y ordenamiento del material y durante las colectas efectuadas; así como a Robert Ortiz, Peter Sánchez, Rosa Rodríguez, Cristian Marte y Hodali Almonte.

Muy agradecido a Giraldo Alayón García (Museo Nacional de Historia Natural de La Habana, Cuba) por sus valiosos consejos y la revisión del manuscrito. Mi agradecimiento además para Altagracia Espinosa (UASD), Sésar Rodríguez (CAD), Jorge Luis Brocca (SOH) y Eladio Fernández (SOH) por su apoyo en la consecución de fondos y en las colectas durante este y otros viajes a la República Dominicana.

\section{LITERATURA CITADA}

Alayón García, G. 1992. La familia Selenopidae (Arachnida: Araneae) en República Dominicana. Poeyana 419: 1-10.

Alayón García, G. 1995a. Adiciones a la familia Prodidomidae (Arachnida: Araneae) en Cuba. Poeyana 451:1-7.

Alayón García, G. 1995b. Nuevo género de Agelenidae (Arachnida: Araneae) de República Dominicana. Poeyana 450: 1-8. 
Alayón García, G. 2002a. Nueva especie de Odo Keyserling (Araneae: Zoridae) de República Dominicana. Revista Ibérica Aracnología 5: 29-32.

Alayón García, G. 2002b. Las arañas endémicas de Cuba (Arachnida: Araneae). Revista Ibérica de Aracnología 2: 1-48.

Alayón García, G. 2004. Notas sobre la familia Ctenidae (Arachnida: Araneae) en La Hispaniola, con la descripción de tres nuevas especies. Revista Ibérica de Aracnología 9: 277-283.

Alayón García, G. 2007. Especie nueva de Pozonia (Araneae: Araneidae) para República Dominicana. Solenodon 6: 41-44.

Alayón García, G., L. F. De Armas \& A. J. Abud Antun. 2001. Presencia de Cyrtophora citricola (Förskal) (Araneae: Araneidae) en las Antillas. Revista Ibérica de Aracnología 4: 9-10.

Ausserer, A. 1875. Zweiter Beitrag zur Kenntniss der Arachnidenfamilie der Territelariæ Thorell (MygalidæAutor). Verh. Zool. Bot. Ges. Wien 25: 125-206.

Banks, N. 1903. A list of the Arachnida from Haiti, with descriptions of new species. Proc. Acad. Nat. Sciences Philadelphia 55:340-345.

Berman, J. D. y H. W. Levi. 1971. The orbweaver genus Neoscona (Araneidae). Bull. Mus. Comp. Zool. 141:465-500.

Bryant, E. B. 1943. The salticid spiders of Hispaniola. Bull. Mus. Comp. Zool. 92: 445-522.

Bryant, E. B. 1945. The Argiopidae of Hispaniola. Bull. Mus. Comp. Zool. 95: 357-418.

Bryant, E. B. 1948. The spiders of Hispaniola. Bull. Mus. Comp. Zool. 100: 332-447.

Chickering, A. 1967. The genus Nops (Araneae, Caponiidae) in Panama and the West Indies. Brev. Mus. Comp. Zool. 272: 1-19.

Coyle, F. A. 1995. A revision of the funnelweb mygalomorph spider subfamily Ischnothelinae (Araneae, Dipluridae). Bull. Amer. Mus. Nat. Hist. 226: 1-133.

Exline, H. y H. W. Levi 1962. American spiders of the genus Argyrodes (Araneae: Theridiidae). Bull. Mus. Comp. Zool. 127: 75-204.

Gertsch, W. J. 1941. Report on some Arachnida from Barro Colorado Island. Amer. Mus. Novit., 1146: 114.

Gertsch, W. J. y F. Ennik 1983. The spider genus Loxosceles in North America, Central America and the West Indies (Araneae, Loxoscelidae). Bull. Amer. Mus. Nat. Hist. 175: 264-360.

Hormiga, G., F. Alvarez-Padilla y S. P. Benjamin. 2007. First records of extant Hispaniolan spiders of the families Mysmenidae, Symphytognathidae, and Ochyroceratidae (Araneae), including a new species of Ochyrocera. American Museum Novitates 3577: 1-21.

Huber, B. A. 2000. New World pholcid spiders (Araneae: Pholcidae): A revision at generic level. Bull. Amer. Mus. Nat. Hist. 254: 1-348.

Huber, B.A. y J. Wunderlich. 2006. Fossil and extant species of the genus Leptopholcus in the Dominican Republic, with the first cases of egg-parasitism in pholcid spiders (Araneae: Pholcidae). Journal of Natural History 40: 2341-2360.

Levi, H. W. 1954. The spider genus Theridula in North and Central America and the West Indies (Araneae: Theridiidae). Trans. Amer. Microsc. Soc. 73: 331-343. 
Levi, H. W. 1955. The spider genera Episinus and Spintharus from North America, Central America and the West Indies (Araneae: Theridiidae). J. New York Entomol. Soc. 62: 65-90.

Levi, H. W. 1957. The spider genera Chrysso and Tidarren in America (Araneae: Theridiidae). J. New York Entomol. Soc. 63: 59-81.

Levi, H. W. 1959. The spider genera Achaearanea, Theridion and Sphyrotinus from Mexico, Central America, and the West Indies (Araneae, Theridiidae). Bull. Mus. Comp. Zool. 121: 55-163.

Levi, H. W. 1963a. The American spider genera Spintharus and Thwaitesia. Psyche 70: 223-234.

Levi, H. W. 1963b. The American spiders of the genus Theridion (Araneae, Theridiidae). Bull. Mus. Comp. Zool. 129: 483-592.

Levi, H. W. 1964. The spider genus Thymoites in America (Aranae: Theridiidae). Bull. Mus. Comp. Zool. 130: 445-471.

Levi, H. W. 1977. The American orb-weaver genera Cyclosa, Metazygia and Eustala North of Mexico (Araneae, Araneidae). Bull. Mus. Comp. Zool. 148: 61-127.

Levi, H. W. 1986. The Neotropical orb-weaver genera Chrysometa and Homalometa (Araneae: Tetragnathidae). Bull. Mus. Comp. Zool. 151:91-215.

Levi, H. W. 1991a. The Neotropical and Mexican species of the orb-weaver genera Araneus, Dubiepeira new genus, and Aculepeira (Araneae: Araneidae). Bull. Mus. Comp. Zool. 152: 167-315.

Levi, H. W. 1991b. The Neotropical orb-weaver genera Edricus and Wagneriana (Araneae: Araneidae). Bull. Mus. Comp. Zool. 152:363-415.

Levi, H. W. 1995. The Neotropical orbweaver genus Metazygia (Araneae: Araneidae). Bull. Mus. Comp. Zool. 154: 63-151.

Levi, H. W. 1999. The Neotropical and Mexican orb weavers of the genera Cyclosa and Allocyclosa (Araneae:Araneidae). Bull. Mus. Comp. Zool. 155:299-379.

Levi, H. W. 2001. The orb weavers of the genera Molinaranea and Nicolepeira, a new species of Parawixia, and comments on orb weavers of temperate South America (Araneae: Araneidae). Bull. Mus. Comp. Zool. 155: 445-475.

Muma, M. H. 1953. A study of the spider family Selenopidae in North America, Central America, and the West Indies. Amer. Mus. Novit. 1629: 1-55.

Peckham, G. W. y E. G. Peckham.1909. Revision of the Attidae of North America. Trans. Wiscons. Acad. Sci. Arts Let. 16(1): 355-655.

Penney, D. 1999. Hypotheses for the Recent Hispaniolan spider fauna based on the Dominican Republic amber spider fauna. Journal of Arachnology 27: 64-70.

Penney, D. 2004. New extant and fossil Dominican Republic spider records, with two new synonymies and comments on taphonomic bias of amber preservation. Revista Ibérica de Aracnología 9: 183-190.

Penney, D. Y D.E. Pérez-Gelabert. 2002. Comparison of the Recent and Miocene Hispaniolan spider faunas. Revista Ibérica de Aracnología 6: 203-223.

Petrunkevitch, A. 1911. A synonymic index-catalogue of spiders of North, Central and South America with all adjacent islands, Greenland, Bermuda, West Indies, Terra del Fuego, Galapagos, etc. Bull. Amer. Mus. Nat. Hist. 29: 1-791. 
Petrunkevitch, A. 1928. The Antillean spider fauna, a study in geographic isolation. Science 68: 650.

Platnick, N. I. 2007. The world spider catalog, version 7.5. American Museum of Natural History, disponible en: http://research.amnh.org/entomology/spiders/catalog/index.html.

Platnick, N. I. y M. U. Shadab 1974. A revision of the bispinosus and bicolor groups of the spider genus

Trachelas in North and Central America and the West Indies. Amer. Mus. Novitates 2560: 1-34.

Platnick, N. I. y M. U. Shadab. 1976. A revision of the spider genera Lygromma and Neozimiris (Araneae, Gnaphosidae). Amer. Mus. Novitates 2598: 1-23.

Platnick, N. I. Y M. U. Shadab 1980. A revision of the spider genus Cesonia (Araneae, Gnaphosidae). Bull. Amer. Mus. Nat. Hist. 165: 335-386.

Sánchez-Ruiz, A. 2005. Una nueva especie de Nops MacLeay, 1839 (Araneae, Caponiidae) de República Dominicana. Revista Ibérica de Aracnología 11:23-27.

Simon, E. 1888. Etudes arachnologiques. 21e mémoire. XXIX. Descriptions d'especes et de genres nouveaux de l'Amerique centrale et des Antilles. Ann. Soc. Ent. France 8: 202-216.

Simon, E. 1895. Histoire Naturelle des Araignées Volume 1, part 4. Paris.

Simon, E. 1903. Histoire Naturelle des Araignées, Volume 2, part 4. Paris.

Strand, E. 1909. Neue oder wenig bekannte südamerikanische Cupiennius- und Ctenus-Arten. Zool. Jahrb. Syst. 28: 293-328.

Strand, E. 1916. Systematische-faunistiche Studien über paläarktische, afrikanische und amerikanische Spinnen des Senckenbergischen Museums. Arch. Naturg. 81(A9): 1-153. 